\title{
Labor Complicated by Cervical Stenosis Following a Laser Cone Biopsy
}

\author{
Jonathan Y. Han ${ }^{\mathrm{a}, \mathrm{e}}$, Wai Loong Wong ${ }^{\mathrm{b}}$, Jerry K.Y. Chan ${ }^{\mathrm{c}, \mathrm{d}}$
}

\begin{abstract}
A 34-year-old lady, with three previous spontaneous vaginal deliveries, presented in labor at $37+0$ weeks' gestation. Following the birth of her third child, she underwent a laser cone biopsy for cervical intraepithelial neoplasia (CIN) 3. Despite sustained regular contractions and augmentation with Syntocinon, progressive cervical dilatation beyond $1 \mathrm{~cm}$ failed to occur in this multiparous lady. A delayed diagnosis of cervical stenosis was made. She eventually underwent a cesarean section where her internal cervical os was found to be thin and fibrotic. This case describes an uncommon occurrence of cervical stenosis presenting in labor and seeks to increase awareness of this condition so as to allow preemptive counselling of similar patients, early recognition in a labor with poor progress and a swift, better-informed decision to deliver via an emergency cesarean section. We review the existing literature on cervical stenosis following cervical conization and analyze the various definitions available.
\end{abstract}

Keywords: Cervical stenosis; Laser cone biopsy; Conization; Complications; Labor dystocia

\section{Introduction}

Local excisional treatment for cervical intraepithelial neoplasia (CIN) or early cervical cancer has been well-known to increase the risk of adverse obstetric outcomes specifically

Manuscript submitted October 8, 2020, accepted October 15, 2020

Published online November 18, 2020

aDepartment of Obstetrics and Gynecology, KK Women's and Children's Hospital, Singapore, Singapore

bDepartment of Gynecological Oncology, KK Women's and Children's Hospital, Singapore, Singapore

'Department of Reproductive Medicine, KK Women's and Children's Hospital, Singapore, Singapore

${ }^{\mathrm{d} C a n c e r}$ and Stem Cell Biology, Duke-NUS Medical School, Singapore, Singapore

${ }^{\mathrm{e}}$ Corresponding Author: Jonathan Y. Han, Department of Obstetrics and Gynecology, KK Women's and Children's Hospital, 100 Bukit Timah Road, Singapore 229899, Singapore. Email: jonathan.han@mohh.com.sg

doi: https://doi.org/10.14740/jmc3605 cervical incompetence, late miscarriages, preterm delivery and preterm premature rupture of membranes (PPROM) $[1,2]$. As preinvasive cervical disease commonly occurs in women of reproductive age, various management strategies have been described to potentially avoid these sequalae [3]. Conversely, scarred cervical stenosis can occur and complicate labor or even result in infertility. We describe an uncommon occurrence of cervical stenosis presenting in labor and resulting in failure to progress in a multiparous lady who had undergone a laser cone biopsy after three previous spontaneous vaginal deliveries.

\section{Case Report}

A 34-year-old patient, gravida six para three, presented to our delivery suite at $37+0$ weeks' gestation in labor. She had two terminations of pregnancies, and three normal vaginal deliveries at $39+0,36+4$, and $38+6$ weeks' gestation in 2005, 2008, and 2016 respectively. Her babies were healthy and weighed between 2,500 to 2,925 g at birth. In May 2017, she underwent a laser cone biopsy for CIN 3 with clear margins achieved. Excisional biopsy was the treatment of choice as it was a type two transformational zone. A laser cone was performed instead of a loop electrosurgical excisional procedure (LEEP) in view of a large transformational zone. Subsequent follow up cervical smear and examination were normal. She had no other significant past medical history.

She conceived spontaneously in 2019 and presented at eight weeks gestation at her booking visit. A transvaginal cervical length scan, performed at 12 weeks' gestation in view of her previous laser cone biopsy, estimated her cervical length to be 3.6 $-3.7 \mathrm{~cm}$. She declined Down syndrome screening and her fetal anomaly scan at 20 weeks' gestation was normal except for an aberrant right subclavian artery. Her pregnancy progressed uneventfully.

The patient presented at 37 weeks with spontaneous rupture of membranes followed by regular contraction pains. Speculum examination confirmed the presence of a definite pool of clear liquor but her cervical os was closed on vaginal examination. She was started on a Syntocinon infusion to augment labor, titrated to maintain her contractions every 2 - 3 min. Her cervix remained closed after $6 \mathrm{~h}$ and a decision was made to stop her Syntocinon infusion and insert a 3 mg dinoprostone (Prostin E2) tablet. A vaginal examination performed $6 \mathrm{~h}$ later revealed a full effaced and $1 \mathrm{~cm}$ dilated 
Table 1. Difference in Diagnostic Criteria for Cervical Stenosis Amongst Various Papers

\begin{tabular}{ll}
\hline Paper & Definition of cervical stenosis \\
\hline $\begin{array}{l}\text { Houlard et al, 2002 [7] } \\
\text { Baldauf et al, 1996 [4] }\end{array}$ & $\begin{array}{l}\text { Cervical os narrowing preventing the insertion of a cotton swab measuring } 4 \mathrm{~mm} . \\
\text { Cervical narrowing that prevented insertion of a 2.5-mm Hegar dilator. Stenosis } \\
\text { was considered complete if there was total occlusion of the cervical canal. }\end{array}$ \\
$\begin{array}{l}\text { Mathevet et al, } 1994 \\
\text { [6], 2003 [10] }\end{array}$ & $\begin{array}{l}\text { Junction not seen (even with an endocervical retractor) or failed calibration with Hegar dilator } 3 \text { mm. } \\
\text { Suh-Burgmann et al, 2000 [9] }\end{array}$ \\
$\begin{array}{l}\text { Munireddy et al, 2012 [11] } \\
\text { Manual dilation was required to allow endocervical sampling with an endocervical curette } 3 \text { mm wide. }\end{array}$ \\
\hline
\end{tabular}

cervix. She was restarted on Syntocinon infusion thereafter but made no progress after $6 \mathrm{~h}$ of augmentation, with contractions felt and seen every $3 \mathrm{~min}$. After discussion with the patient, another dinoprostone tablet was inserted, and Syntocinon augmentation recommenced $6 \mathrm{~h}$ later. However, after another $4 \mathrm{~h}$, there was no change to her cervical findings despite regular contractions every $2-3$ min. A diagnosis of cervical stenosis was made.

She underwent an emergency cesarean section for failure to progress and delivered a healthy baby girl with a birthweight of 3,515 $\mathrm{g}$ and an estimated blood loss of $400 \mathrm{~mL}$. Intraoperatively, her internal cervical os was found to be one cm dilated, thin and fibrotic. She had an uncomplicated recovery and was discharged well on the third postoperative day.

\section{Discussion}

Several definitions of cervical stenosis have been suggested with no widely agreed consensus, with the incidence of cervical stenosis following laser conization ranging from $7.0-16.8 \%$ [4-7]. In an early study of patients treated by cold knife conization between 1968 and 1973 by Moinian et al [8], six out of 100 full-term pregnancies ended up with cesarean delivery due to failure to dilate due to "rigid scarred cervix". Houlard et al later showed that up to $16.8 \%$ of women could develop cervical stenosis post laser cone biopsy, as defined by the inability to pass a $4 \mathrm{~mm}$ cotton swab through the cervical os, with older aged women being at higher risk, as well as larger lesions and depth of excision, endocervical lesions and the use of continuous laser mode [7]. Other studies have suggested lower risks of cervical stenosis, but the definition had been heterogenous (Table 1) [4, 6, 7, 9-11]. In the largest meta-analysis of observational and cohort studies of 65,082 treated and 6,292,563 untreated patients, there were no differences found in cervical stenosis or cesarean delivery rates [1].

A study by Mathevet et al looked into the outcomes between the three techniques of conization and found that cervical stenosis occurred in $29 \%$ of women who underwent a cold knife cone biopsy, compared to $9 \%$ and $19 \%$ in the laser and LEEP categories respectively, however the data was not statistically significant [6]. In the subsequent long-term follow-up study [10], two women required cesarean sections for cervical dystocia; one was previously treated with laser conization while the other with LEEP. Mathevet et al $[6,10]$ reported that these findings, although from a small sample size, appeared to be consistent with an earlier study by Leiman et al in 1980 , which suggested that larger cones sizes are associated with increased risks of preterm complications, while smaller cones sizes are associated with cervical stenosis resulting in cesarean section [12].

Aside from Moinian et al's study in the late 60 s to early 70 s regarding diagnosis of cervical stenosis only at labor [8], there is one other case report by Munireddy et al [11] where cervical stenosis prevented any cervical dilatation in a primiparous lady treated with a laser cone biopsy 6 years beforehand. In our multiparous patient, her cervical stenosis was a complication from her previous laser cone biopsy. This was not diagnosed since she had normal menses and was able to conceive naturally. Despite sustained regular contractions and augmentation with Syntocinon, progressive cervical dilatation beyond $1 \mathrm{~cm}$ failed to occur in this multiparous lady, leading to a delayed diagnosis, and eventual cesarean delivery.

\section{Conclusions}

Counselling of patients, especially those of child-bearing age, regarding local excisional treatment for preinvasive cervical disease should include the risks of cervical stenosis and future adverse obstetric sequalae. The patient's desire for future fertility must be explored and taken into account before deciding on the mode of treatment. As obstetric complications range widely from cervical insufficiency resulting in preterm delivery or PPROM to cervical stenosis resulting in infertility or failure to progress in labor, close antenatal surveillance is paramount in subsequent pregnancies and should involve a multidisciplinary team comprising obstetricians, gynaecologic oncologists, maternal fetal specialists and neonatologists. Understanding the potential for cervical stenosis to occur, although uncommon, would allow preemptive counselling of these patients when they present in labor, early recognition in a labor with poor progress and a swift, better-informed decision to deliver via an emergency cesarean section.

\section{Acknowledgments}

The authors would like to acknowledge the valuable support and input of the delivery suite nurses, midwifes and doctors of KK Women's and Children's Hospital, Singapore, Singapore. 


\section{Financial Disclosure}

JCKY received salary support from Singapore's Ministry of Health's National Medical Research Council (CSASI-008-2016). The authors have not declared a specific grant for this research from any funding agency in the public, commercial or not-for-profit sectors.

\section{Conflict of Interest}

None to declare.

\section{Informed Consent}

Informed consents have been obtained from the patient for publication.

\section{Author Contributions}

All authors were involved in the clinical care of the patient. JH collected the relevant data and drafted the manuscript. WL Wong was involved in editing the manuscript. JC was the obstetrician in charge of the patient and was involved in the conception and editing of the manuscript.

\section{Data Availability}

The authors declare that data supporting the findings of this study are available within the article.

\section{References}

1. Kyrgiou M, Athanasiou A, Kalliala IEJ, Paraskevaidi M, Mitra A, Martin-Hirsch PP, Arbyn M, et al. Obstetric outcomes after conservative treatment for cervical intraepi- thelial lesions and early invasive disease. Cochrane Database Syst Rev. 2017;11:CD012847.

2. Sjoborg KD, Vistad I, Myhr SS, Svenningsen R, Herzog C, Kloster-Jensen A, Nygard G, et al. Pregnancy outcome after cervical cone excision: a case-control study. Acta Obstet Gynecol Scand. 2007;86(4):423-428.

3. Jolley JA, Wing DA. Pregnancy management after cervical surgery. Curr Opin Obstet Gynecol. 2008;20(6):528533.

4. Baldauf JJ, Dreyfus M, Ritter J, Meyer P, Philippe E. Risk of cervical stenosis after large loop excision or laser conization. Obstet Gynecol. 1996;88(6):933-938.

5. Bostofte E, Berget A, Falck Larsen J, Hjortkjaer Pedersen P, Rank F. Conization by carbon dioxide laser or cold knife in the treatment of cervical intra-epithelial neoplasia. Acta Obstet Gynecol Scand. 1986;65(3):199-202.

6. Mathevet P, Dargent D, Roy M, Beau G. A randomized prospective study comparing three techniques of conization: cold knife, laser, and LEEP. Gynecol Oncol. 1994;54(2):175-179.

7. Houlard S, Perrotin F, Fourquet F, Marret H, Lansac J, Body G. Risk factors for cervical stenosis after laser cone biopsy. Eur J Obstet Gynecol Reprod Biol. 2002;104(2):144-147.

8. Moinian M, Andersch B. Does cervix conization increase the risk of complications in subsequent pregnancies? Acta Obstet Gynecol Scand. 1982;61(2):101-103.

9. Suh-Burgmann EJ, Whall-Strojwas D, Chang Y, Hundley D, Goodman A. Risk factors for cervical stenosis after loop electrocautery excision procedure. Obstet Gynecol. 2000;96(5 Pt 1):657-660.

10. Mathevet P, Chemali E, Roy M, Dargent D. Longterm outcome of a randomized study comparing three techniques of conization: cold knife, laser, and LEEP. Eur J Obstet Gynecol Reprod Biol. 2003;106(2):214-218.

11. Raja Appireddyhalli Munireddy, Burnham Annabelle. Cervical Stenosis following a laser cone biopsy - an uncommon presentation in labour. Journal of Clinical and Diagnostic Research. 2012;6(7):1306-1307.

12. Leiman G, Harrison NA, Rubin A. Pregnancy following conization of the cervix: complications related to cone size. Am J Obstet Gynecol. 1980;136(1):14-18. 